\title{
Standard Heterosis for De-husked Cob Yield in Baby Corn (Zea mays L.) Hybrids
}

\author{
Sandeep Kumar Bangarwa* and R. B. Dubey
}

Department of Genetics and Plant Breeding, Rajasthan College of Agriculture, Maharana Pratap University of Agriculture and Technology, Udaipur-313001 (Raj.), India

*Corresponding author

Keywords

Baby Corn, Zea mays L., Hybrids

\section{Article Info}

Accepted:

20 December 2020

Available Online:

10 January 2021

\begin{abstract}
A B S T R A C T
The present investigation consisted of a total of 45 hybrids along with 18 parents (15 inbred lines and 3 testers) and 2 checks. A total of 65 entries were evaluated in randomized block design with three replications over three locations (Two during Kharif-2019 and one during Rabi 2019-20) at Instructional farm, Rajasthan College of Agriculture, Udaipur, Rajasthan (Kharif-2019 and Rabi 2019-2020) and Agriculture Research Sub Station, Vallabh Nagar, Udaipur, Rajasthan (Kharif-2019). Observations were recorded on eighteen characters for estimates of the extent of standard heterosis. The perusal of estimates of standard heterosis for de-husked cob yield and its contributing characters revealed that on pooled basis, maximum and significantly desirable economic heterosis was depicted by cross EIQ-103 x EI-2156 (13.51). The cross combinations viz., EIQ-103 × EI-2156 (13.51), EIQ-180 × EI-2156 (16.02), EIQ-103 × EI-2156(10.54), EIQ-103 × EI2156 (17.26), EIQ-180 × EI-2156 (9.46) were identified suitable for yield and yield contributing traits.
\end{abstract}

\section{Introduction}

Maize (Zea mays L.) $2 \mathrm{n}=20$, is the third most important cereal crop after rice and wheat in the world. By origin, maize is native to South America. It is grown during rainy (Kharif), winter Rabi and spring seasons, but major production is in the Kharif rainy season. It has diversified uses as food for humans, feed for livestock and raw material for industries. It has immense potential, and is, therefore, called the "miracle crop". Maize being a C4 plant, is an efficient converter of absorbed nutrients into food. Globally, maize is known as 'queen of cereals' because of its highest genetic yield potential. It is the only food cereals crop that can be grown in diverse seasons, ecologies and uses. Beside this maize have many types like normal yellow/white grain, sweet corn, baby corn, popcorn, waxy corn, high amylose corn, high oil corn, quality protein maize etc. Apart from this, maize is an important industrial raw material and provides large opportunity for value addition.

A recent trend is of growing maize for vegetable purpose, which is commonly known as 'Baby corn'. Baby corn is a young 
finger like unfertilized cob of maize harvested early within 1-3 days of silk emergence. Baby corn is a good option for crop diversification (Dass et al., 2008) and it suits to periurban agriculture.

Baby corn is a delicious, decorative and nutritious vegetable, without cholesterol. It is a low caloric vegetable which is rich in fibre content. One baby corn can be compared with an 'egg' in terms of minerals. Nutritive values of baby corn (per $100 \mathrm{~g}$ of edible portion) is Moisture 89.10 \%, Carbohydrates 8.20 g, Protein $1.90 \mathrm{~g}$, Calcium $28.00 \mathrm{mg}$, Phosphorus $86.00 \mathrm{mg}$, Iron $0.10 \mathrm{mg}$. (Jat et al., 2019)

Worldwide, Thailand is the leading producer and exporter of baby corn. India is emerging as the potential producer of baby corn due to high demand with less cost of production. In India, baby corn is being cultivated in Meghalaya, Western Uttar Pradesh, Haryana, Maharashtra, Karnataka and Andhra Pradesh.

Heterosis, the measure of the average superiority of a hybrid over its parental inbred lines is an important consideration in the development of hybrid varieties.

Diversity among inbred source populations is an important factor in determining combining ability among inbred lines and heterosis revealed by hybrids, where a more diverse combination is expected to produce more superior hybrids (Prasad and Singh, 1986).

Despite manifold uses of baby corn, very little information is so far available on standard heterosis in baby corn.

In view of the above facts, the present investigation is employed to derive information on standard heterosis.

$\mathrm{F} 1=$ Mean value of hybrid

\section{Materials and Methods}

The present investigation consisted of a total of 45 hybrids along with 18 parents (15 inbred lines and 3 testers) and 2 checks. A total of 65 entries were evaluated in randomized block design with three replications over three locations (Two during Kharif-2019 and one during Rabi 2019-20) at Instructional farm, Rajasthan College of Agriculture, Udaipur, Rajasthan (Kharif-2019 and Rabi 2019-2020) and Agriculture Research Sub Station, Vallabh Nagar, Udaipur, Rajasthan (Kharif-2019). Observations were recorded on eighteen characters for estimates of the extent of standard heterosis. The experimental material comprised of 15 inbred lines viz.,EI-2311-4, EI-2449-2, EI-2403, EIQ-103, EIQ-104, EI1104-1, EI-561-2, EI-2173, EI-2177-2, EI2509, EI-2518-2, EI-11-3, EIQ-180, EIQ-225, EIQ-235 and 3 testers EI-670-2, EI-2518-4, EI-2156 their $45 \mathrm{~F}_{1 \mathrm{~s}}$ and two checks viz., HM4 and VL Baby Corn-2. These $45 \mathrm{~F}_{1 \mathrm{~s}}$ were obtained by crossing 15 inbred lines and 3 testers in line $\times$ tester mating design.

Economic heterosis were calculated according to the method suggested by Meredith and Bridge (1972).

\section{Economic heterosis}

Economic heterosis $(\%)=\frac{\left(\overline{F_{1}}-\overline{B C}\right)}{\overline{B C}} \times 100$

It's significance was tested by using student " $t$ " test.

$$
\begin{gathered}
t_{E D F}=\frac{\overline{F_{1}}-\overline{B C}}{S E\left(\overline{F_{1}}-\overline{B C}\right)} \\
S E_{\left(\overline{F_{1}}-\overline{B C}\right)}=\sqrt{\frac{2 M S E}{n}}
\end{gathered}
$$

Where, 


\begin{tabular}{|l|l|l|}
\hline BC & $=$ & Mean value of best checks \\
\hline $\mathbf{N}=$ & $\begin{array}{l}\text { Divisor in respective conditions i.e. } \mathrm{r} \text { in } \\
\text { case of individual environment and } \mathrm{rs} \text { in } \\
\text { over the environments. }\end{array}$ \\
\hline
\end{tabular}

\begin{tabular}{|l|l|l|l|}
\hline MSE & $=\begin{array}{l}\text { Error mean square from for } \\
\text { individual over } \\
\text { environments, respectively. }\end{array}$ & $\begin{array}{l}\text { for } \\
\text { the }\end{array}$ \\
\hline $\mathbf{t}_{\mathrm{EDF}}=$ & Student's ' $\mathrm{t}$ ' at error degree of freedom \\
\hline
\end{tabular}

\section{Results and Discussion}

Maximum significant economic heterosis for de-husked cob yield per plant was recorded in one cross viz., EIQ-103 × EI-2156 (13.51) on pooled basis. The significant economic heterosis varied from cross EIQ-103 x EI2156 (13.51) to EI-561-2 × EI-670-2 (-14.4). Two crosses showed significantly desirable economic heterosis for husked cob yield per plant. Maximum significantly desirable economic heterosis was observed in cross EIQ-103 × EI-2156 (17.26) followed by EIQ$180 \times$ EI-2156(16.68) for husked cob yield per plant on over the environments. The significant economic heterosis ranged from EIQ-103 $\times$ EI-2156 (17.26) to EI-561-2 × EI670-2 (-14.17) for this trait on pooled basis. Four crosses showed significantly desirable economic heterosis for husked cob weight on pooled basis. Maximum significantly desirable positive economic heterosis was depicted by cross EIQ-180 × EI-2156 (16.02) followed by EIQ-103 × EI-2156(10.54), EIQ$180 \times$ EI-2518-4(9.84) and EI-1104-1 $\times$ EI670-2(8.48) for husked cob weight on over the environments. On pooled basis the standard heterosis varied from 16.02 (EIQ$180 \times$ EI-2156) to $-11.67($ EI-2311-4 $\times$ EI2518-4) for this trait. Eight crosses depicted significant economic heterosis on pooled basis. None of the cross depicted significantly desirable positive economic heterosis for dehusked cob weight on pooled basis. The significant standard heterosis varied from $9.66($ EI-2311-4 × EI-670-2, EIQ-104 × EI-
670-2) to -16.25 (EI-2311-4 × EI-2518-4) for this trait. The perusal of Table 5.2.1 indicated among the characters, maximum numbers of crosses with significant desirable standard heterosis were found for days to first and last cob picking interval followed by crude protein content.

Maximum significant economic heterosis for de-husked cob yield per plant was recorded in one cross viz., EIQ-103 × EI-2156 (13.51) on pooled basis. Four crosses showed significantly desirable economic heterosis for husked cob weight on pooled basis. Maximum significantly desirable positive economic heterosis was depicted by cross EIQ-180 $\times$ EI-2156 (16.02) followed by EIQ$103 \times$ EI-2156(10.54), EIQ-180 × EI-25184(9.84) and EI-1104-1 × EI-670-2(8.48) for husked cob weight on over the environments. Two crosses depicted significantly desirable economic heterosis for husked cob yield per plant.

Maximum significantly desirable economic heterosis was observed in cross EIQ-103 $\times$ EI-2156 (17.26) followed by EIQ-180 × EI2156(16.68) for husked cob yield per plant on over the environments. Eight crosses depicted significantly desirable economic heterosis for plant height on pooled basis. Maximum economic heterosis was observed in EIQ-180 $\times$ EI-670-2 (10.61) followed by EI-2177-2 $\times$ EI-2156 (10.04) and EI-561-2 $\times$ EI-670-2 (9.85) for plant height on over the environments.

Two crosses (EI-2311-4 $\times$ EI-2156 and EI2177-2 × EI-2518-4) depicted significantly desirable economic heterosis for moisture content. Maximum significantly positive economic heterosis was depicted by cross EI2177-2 × EI-2518-4 (3.82) followed by EI2311-4 $\times$ EI-2156 (3.38) on pooled basis for moisture content. 
Table.1 Analysis of variance on over the environments

\begin{tabular}{|c|c|c|c|c|c|c|c|c|c|c|c|c|c|c|c|c|c|c|c|}
\hline \multirow[b]{2}{*}{$\begin{array}{l}\text { Source of } \\
\text { variation }\end{array}$} & \multirow[b]{2}{*}{ df } & \multicolumn{18}{|c|}{ Mean sum of squares } \\
\hline & & 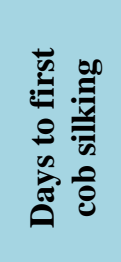 & 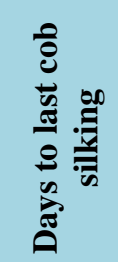 & 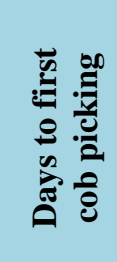 & 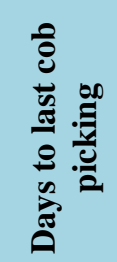 & 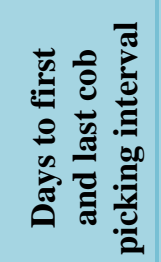 & 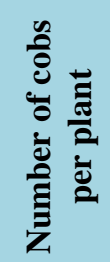 & 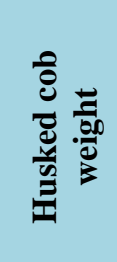 & 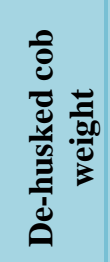 & 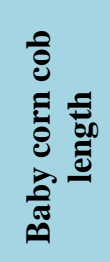 & 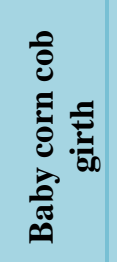 & 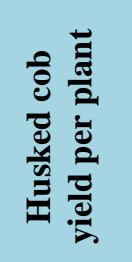 & 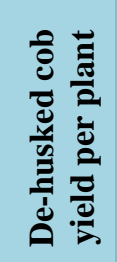 & 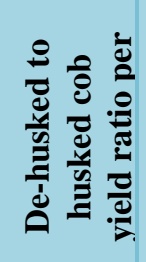 & 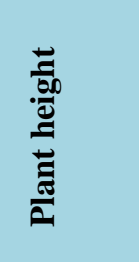 & 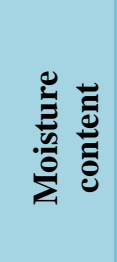 & 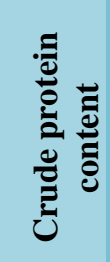 & $\mathscr{2}$ & 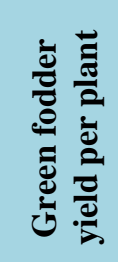 \\
\hline Environments & 2 & $\begin{array}{l}15990 \\
.87 * *\end{array}$ & $\begin{array}{c}29353 \\
.3 * *\end{array}$ & $\begin{array}{l}18747 \\
.19 * *\end{array}$ & $\begin{array}{l}36933 \\
.13^{* *}\end{array}$ & $\begin{array}{c}3189.23 \\
* *\end{array}$ & $\begin{array}{c}12.22 \\
* *\end{array}$ & $\begin{array}{l}2371 . \\
73^{* *}\end{array}$ & $\begin{array}{l}85.9 \\
8 * *\end{array}$ & $\begin{array}{c}143.4 \\
4 * *\end{array}$ & $\begin{array}{l}78.9 \\
9 * *\end{array}$ & $\begin{array}{c}74304 . \\
18 * *\end{array}$ & $\begin{array}{l}2655 . \\
61 * *\end{array}$ & $\begin{array}{c}0.0115 \\
* *\end{array}$ & $\begin{array}{c}20306.2 \\
3 * *\end{array}$ & $\begin{array}{c}178.8 \\
* *\end{array}$ & $\begin{array}{l}184 . \\
63 * *\end{array}$ & $\begin{array}{c}1.4 \\
1\end{array}$ & $\begin{array}{l}131228 \\
1.29 * *\end{array}$ \\
\hline Treatments & 62 & $\begin{array}{c}20.2^{*} \\
*\end{array}$ & 26.13 & $\begin{array}{c}13.45 \\
* *\end{array}$ & $\underset{* *}{11.19}$ & $12.18 * *$ & $0.2 * *$ & $\begin{array}{c}202.8 \\
4 * *\end{array}$ & $\begin{array}{c}5.06 \\
* *\end{array}$ & $\begin{array}{c}4.66^{*} \\
*\end{array}$ & $\begin{array}{c}1.01 \\
* *\end{array}$ & $\begin{array}{c}2643.6 \\
7 * *\end{array}$ & $\begin{array}{c}72.44 \\
* *\end{array}$ & $\begin{array}{c}0.0003 \\
* *\end{array}$ & $\begin{array}{c}- \\
9966.19\end{array}$ & $\begin{array}{l}9.34 * \\
*\end{array}$ & $\begin{array}{c}3.26 \\
* *\end{array}$ & $\begin{array}{l}3.3 \\
5^{* *}\end{array}$ & $\begin{array}{c}94230 . \\
54 * *\end{array}$ \\
\hline $\begin{array}{l}\text { Treatments x } \\
\text { Environments }\end{array}$ & 124 & $\begin{array}{c}16.9 * \\
*\end{array}$ & $\begin{array}{c}31.49 \\
* *\end{array}$ & $\begin{array}{c}14.4^{*} \\
*\end{array}$ & $\begin{array}{c}11.78 \\
* *\end{array}$ & $12.26 * *$ & $\begin{array}{c}0.13 * \\
*\end{array}$ & $\begin{array}{c}48.99 \\
* *\end{array}$ & $\underset{* *}{1.51}$ & $\begin{array}{c}1.56^{*} \\
*\end{array}$ & $\underset{* *}{0.52}$ & $\begin{array}{c}580.91 \\
* *\end{array}$ & $\begin{array}{c}18.78 \\
* *\end{array}$ & $\begin{array}{c}0.0002 \\
* *\end{array}$ & $\begin{array}{c}2729.29 \\
* *\end{array}$ & $\begin{array}{l}7.72 * \\
*\end{array}$ & $\begin{array}{c}2.86 \\
* *\end{array}$ & $\begin{array}{l}3.7 \\
* *\end{array}$ & $\begin{array}{c}16694 . \\
54 * *\end{array}$ \\
\hline Error & 378 & 6.07 & 21.03 & 2.19 & 2.22 & 2.59 & 0.03 & 5.75 & 0.28 & 0.37 & 0.14 & 102.76 & 4.13 & 0.0001 & 51.93 & 1.92 & 0.24 & $\begin{array}{c}0.5 \\
9\end{array}$ & 779.5 \\
\hline $\operatorname{SEm}(g) \pm$ & & 0.82 & 1.53 & 0.49 & 0.5 & 0.54 & 0.06 & 0.8 & 0.18 & 0.2 & 0.12 & 3.38 & 0.68 & 0 & 2.4 & 0.46 & 0.16 & $\begin{array}{c}0.2 \\
6\end{array}$ & 9.31 \\
\hline $\operatorname{CD}(5 \%)$ & & 2.28 & 4.25 & 1.37 & 1.38 & 1.49 & 0.16 & 2.22 & 0.49 & 0.56 & 0.35 & 9.4 & 1.88 & 0.01 & 6.68 & 1.28 & 0.45 & $\begin{array}{c}0.7 \\
1\end{array}$ & 25.88 \\
\hline $\operatorname{SEm}(g x e) \pm$ & & 1.42 & 2.65 & 0.85 & 0.86 & 0.93 & 0.1 & 1.38 & 0.31 & 0.35 & 0.22 & 5.85 & 1.17 & 0.01 & 4.16 & 0.8 & 0.28 & $\begin{array}{c}0.4 \\
4\end{array}$ & 16.12 \\
\hline $\operatorname{CD}(5 \%)$ & & 3.96 & 7.36 & 2.38 & 2.39 & 2.58 & 0.28 & 3.85 & 0.85 & 0.98 & 0.6 & 16.27 & 3.26 & 0.02 & 11.57 & 2.22 & 0.79 & $\begin{array}{c}1.2 \\
3\end{array}$ & 44.82 \\
\hline CV & & 4.14 & 6.89 & 2.38 & 2.14 & 21.66 & 6.51 & 5.37 & 6.23 & 7.15 & 8.28 & 8.46 & 8.94 & 5.25 & 4.62 & 1.86 & 6.18 & $\begin{array}{c}8.3 \\
7\end{array}$ & 8.27 \\
\hline GM & & 59.49 & 66.53 & 62.13 & 69.55 & 7.43 & 2.66 & 44.69 & 8.49 & 8.51 & 4.52 & 119.8 & 22.73 & 0.19 & 155.97 & 74.64 & 7.93 & $\begin{array}{c}9.1 \\
7\end{array}$ & 337.63 \\
\hline
\end{tabular}

*,** indicates significant at $5 \%$ and $1 \%$, respectively 
Ten crosses showed significantly desirable economic heterosis for crude protein content. Maximum significant positive economic heterosis was depicted by cross EIQ-180 $\times$ EI-2518-4 (19.46) followed by cross EIQ-104 $\times$ EI-670-2 (16.9) and EIQ-180 × EI-670-2 (16.52) for crude protein content on pooled basis. Two crosses depicted significantly desirable economic heterosis for TSS.

The estimates of maximum economic heterosis were depicted by cross EIQ-225 $\times$ EI-670-2 (15.05) followed by EIQ-104 $\times$ EI2518-4 (13.68) for TSS on pooled basis. Six crosses depicted significantly desirable economic heterosis for TSS. Maximum significant positive economic heterosis was observed on over the environments in cross EI-2449-2 × EI-2518-4 (29.05) followed by EI-2449-2 $\times$ EI-670-2 (26.86) and EIQ-235 $\times$ EI-2518-4 (25.20) for green fodder yield per plant (Table-1).

\section{References}

Dass, Sain, Yadav, V. K., Kwatra, A., Jat, M. L., Rakshit, S., Kaul, J., Prakash, O., Singh, I., Singh, K.P. and Sekhar, J. C. 2008. Baby Corn in India. Directorate of Maize Research, Pusa Campus, New Delhi, Technical Bulletin, 6:1-45.

Jat, S.L., Parihar, C.M., Preeti, Radheshyam, Kumar, B., Chikkappa G.K. and Singh, A.K. 2019. Baby corn and sweet corn production technologies for Mizoram. National workshop on Scientific Maize Cultivation in North East India, 5th March 2019, Aizawl, Mizoram: 8-21.

Meredith, W. R. and Bridge, R. R. 1972. Heterosis and gene action in cotton (Gossipium hirsutum). Crop Sci., 12:304-310.

Prasad, S. K. and Singh, T. P. 1986. Heterosis in relation to genetic divergence in maize (Zea mays L.). Euphytica, 35: 919-924.

\section{How to cite this article:}

Sandeep Kumar Bangarwa and Dubey, R. B. 2021. Standard Heterosis for De-husked Cob Yield in Baby Corn (Zea mays L.) Hybrids. Int.J.Curr.Microbiol.App.Sci. 10(01): 3550-3554. doi: https://doi.org/10.20546/ijcmas.2021.1001.419 